\title{
Seeing Sounds and Hearing Sights: The Influence of Prior Learning on Current Perception
}

\author{
Jennifer D. Ryan ${ }^{1,2}$, Sandra N. Moses ${ }^{1}$, Melanie L. Ostreicher ${ }^{1}$, \\ Timothy Bardouille ${ }^{1}$, Anthony T. Herdman ${ }^{3}$, Lily Riggs ${ }^{1,2}$, \\ and Endel Tulving ${ }^{1,2}$
}

\begin{abstract}
It is well known that previous perceptual experiences alter subsequent perception, but the details of the neural underpinnings of this general phenomenon are still sketchy. Here, we ask whether previous experiences with an item (such as seeing a person's face) leads to the alteration of the neural correlates related to processing of the item as such, or whether it creates additional associative connections between such substrates and those activated during prior experience. To address this question, we used magnetoencephalography (MEG) to identify neural changes accompanying subjects' viewing of unfamiliar versus famous faces and hearing the names of unfamiliar versus famous names. We were interested in the nature of the involvement of auditory brain regions in the viewing of faces, and in the involvement of visual regions in the hearing of names. Evoked responses from MEG re-
\end{abstract}

\section{INTRODUCTION}

What we see or hear is determined not only by the sensory stimuli and their context but also by our previous experiences with the stimuli. In Principles of Psychology, William James (1890) enunciated his famous General Law of Perception: "whilst part of what we perceive comes through our senses from the object before us; another part (and it may be the larger part) always comes ... out of our own head." Now, more than a hundred years later, research is outlining how our previous experiences serve to influence and alter the very nature of perception. The idea expressed in this statement is now universally accepted, and a rich literature exists on top-down and bottom-up processes in perception.

How are our perceptions changed by prior events? For instance, imagine that you meet someone for the first time and engage in conversation, and then you meet that person again a month later. Is your perceptual experience of the person's face exactly the same a month later as it was when you first met? If not, what changes? One possibility is that the processing of the face remains the

${ }^{1}$ The Rotman Research Institute, Baycrest, ${ }^{2}$ University of Toronto, ${ }^{3}$ Simon Fraser University, Toronto, Canada cordings for the names and faces conditions were localized to auditory and visual cortices, respectively. Unsurprisingly, peak activation strength of evoked responses was larger for famous versus nonfamous names within the superior temporal gyrus (STG), and was similar for famous and nonfamous faces in the occipital cortex. More relevant to the issue of experience on perception, peak activation strength in the STG was larger for viewed famous versus nonfamous faces, and peak activation within the occipital cortex was larger for heard famous versus nonfamous names. Critically, these experiencerelated responses were present within 150-250 msec of stimulus onset. These findings support the hypothesis that prior experiences may influence processing of faces and names such that perception encompasses more than what is imparted on the senses.

same between both meetings, but the knowledge you have learned about the person becomes available upon viewing the face. Another possibility is that the knowledge you have learned about the person actually changes the way in which you perceive his or her face.

One way to address these questions is to examine neural responses and assess whether such activity can be altered by previous experience during the time at which perception is occurring. Here, we consider "perception" to refer to the construction of a coherent representation regarding sensory input and which can bring to bear stored information regarding the item itself, whereas "perceptual processing" may refer to the underlying mechanisms involved in such construction. The issue here is whether prior learning experiences are coupled with input from the senses to form such a perceptual representation. Indeed, there is some indication that the neural regions associated with perceptual processing are altered by our experiences. For instance, Nyberg, Habib, McIntosh, and Tulving (2000) exposed their subjects to pairs of visually presented words and auditorily presented sounds, and then showed them these words alone, one at a time, while using functional magnetic resonance imaging (fMRI) to look for brain 
activation in visual and auditory regions. Initially, the visual stimuli did not elicit activation within auditory regions. However, by virtue of pairing the words with sounds, the visual presentation of the previously encountered words alone then led to activity in the auditory regions. Similar findings were obtained by Wheeler, Petersen, and Buckner (2000): The pairing of items, each presented in its unique modality, later produced activation in neural regions underlying processing in an item's own presentation modality but also in regions of the associated item's modality. These findings are intriguing, but although these fMRI results illuminate the neural correlates of the venerable phenomenon and concept of association, questions remain regarding the association itself.

The purpose of the study reported here was to probe more deeply into the nature of the kind of "association" demonstrated in the Nyberg et al. (2000) and Wheeler et al. (2000) studies. Does the neural activity in the auditory regions when the subject views a visually presented stimulus signify a neural "connection" (underlying an acquired association or a mental "link") between the previously presented stimuli, the ages-old standard view (e.g., Anderson, 1976)? Or does it perhaps signify a change in the visual item's processing, and a change in how it is now "perceived"? In other words, does it perhaps tell us something about the neural underpinnings of William James's Law of Perception?

Although these fMRI studies can tell us which brain regions are related to associative processing of sensory inputs, they did not throw much light on the issue we are raising here: Can previous experience with an object change the way the object is subsequently "perceived"? Although the above studies demonstrate that the processing of stimuli is altered through prior experience, it is not clear what stage of processing is affected. Is perceptual processing altered through prior experience or is associated information activated following perceptual processing? The current work used a methodology that provides information about the temporal dynamics of the neural connection between the brain regions that represent the association. Such an investigation would illuminate the question we are discussing here, involving "auditory" neural activity upon the presentation of a visual stimulus. If we had information about the time when the auditory region becomes active in relation to the physical presentation of the visual stimulus, and vice-versa, we would be in a better position to evaluate the issue of whether our real-life experiences result in "changed perception" versus "acquired associations." That is, auditory neural activity elicited by a visual stimulus that occurs with the same time course as activity in visual regions may suggest that prior experience alters our perceptual representations. On the other hand, auditory activity elicited by a visual stimulus that follows, in time, activity in visual regions may suggest that our prior experiences are stored as acquired associations and are merely reactivated upon viewing a previously encountered stimulus.
Recently, researchers have provided initial evidence that begins to address this issue. Moses, Martin, Houck, Ilmoniemi, and Tesche (2005) presented their subjects with delay and trace conditioning paradigms in which an auditory noise burst was paired with a visual presentation of a geometric figure. Again, presentation of the visual stimuli alone did not elicit activity within auditory regions, however, following several trials of training in which the noise and visual stimulus were associated, the presentation of the geometric figure alone elicited a response within the auditory cortex. Because the researchers used magnetoencephalography (MEG), which provides superior temporal information compared to fMRI, they were able to discern that this response within the auditory cortex occurred early (approximately $50 \mathrm{msec}$ ) following the offset of the visual stimulus, at the time when the presentation of the noise would have ordinarily been expected. In a further analysis, Moses et al. (2007) noted that following training, early responses were observed in the amygdala within 200 msec following the onset of the visual stimulus that had been previously paired with the noise compared to visual stimuli that had never been paired with the noise. This suggests, at the very least, that the experiences learned by the subjects within the laboratory altered their subsequent neural responses, and possibly, perception of the items.

However, do such early changes in neural activity represent a general and obligatory change in perception? Such findings may merely reflect a temporary change, an artifact of the experiment. Note that the above studies all provided the learning within a single experimental session. The observed changes in neural activity may only be transient changes, which, although present during the learning situation, may not occur at a later date even if the knowledge regarding the link between the noise and the picture can be brought forth. Additionally, the observed neural changes may not reflect an obligatory change in perception because, for some of the above studies (Nyberg et al., 2000; Wheeler et al., 2000), the learned associations could have been explicitly recruited in an effort to perform the tasks that were given to the subjects, thereby resulting in increased activation in associated neural regions. For instance, to recall whether a picture had been previously viewed, it would be advantageous to recall other elements that were present during the time of the original learning episode in order to verify whether one's judgment of "previously studied" versus "novel" is indeed accurate. Definitive evidence that our experiences create changes to perception would require a demonstration that such neural changes are long-lasting and occur even when the associated information may not be relevant for the task at hand. For these reasons, we employed a paradigm here in which the "previous learning episodes" occurred in real life, and in which the learned associated information was not presented at any time during the study and was not needed in order to complete the task we gave to the subjects. 
This study, then, is the first exploration of whether real-life experiences change perception. Like the Moses et al. (2005) study, we used MEG to outline when differences in neural activity could be observed as a result of prior experiences. Subjects viewed faces or heard names of famous and nonfamous people. We examined the extent to which visual regions were activated upon hearing famous versus nonfamous names, and likewise, the extent to which auditory regions were activated upon viewing famous versus nonfamous faces. We did not ask subjects to reflect upon the contents of their memories during the task or to make use of associated information in any way; rather we solely instructed them to judge whether a given face/name was male or female. If our previous experiences, indeed, change our perceptual representations, famous stimuli should elicit early neural responses in regions which are typically activated during the processing of associated information. For instance, viewing a famous face should elicit early activity from regions ordinarily related to the processing of auditory/ verbal information; likewise, hearing a famous name should elicit early activity from regions ordinarily related to the processing of visual/facial information. In order to suggest that prior experiences result in a change in perception, such responses across multiple sensory regions should occur within the time window that is generally assumed to be related to perceptual processing rather than later stages of processing which may include conceptual processing or semantic elaboration. Using findings from the event-related brain potential (ERP) literature as a guide, we suggest that differences in neural activity between famous and nonfamous conditions that occur prior to 300 msec may predominantly reflect changes in perceptual processing rather than changes in postperceptual processes, such as the access to semantic or conceptual information (e.g., Pickering \& Schweinberger, 2003; Schweinberger, Pickering, Burton, \& Kaufmann, 2002), although precise temporal delineation between perceptual and postperceptual processes remains controversial (Itier, Herdman, George, Cheyne, \& Taylor, 2006). Additionally, we would take findings of simultaneous (as opposed to successive) responses within visual and auditory regions for the famous conditions as evidence of altered perceptual representations rather than as the activation of distinct, associated information. To return to the example of meeting someone new, such findings of early neural changes as a result of prior experience would show that learning something about the person behind the face alters the very manner in which you perceive the face when you meet him or her again.

\section{METHODS}

\section{Subjects}

Ten adults (3 men; 9 right-handed), aged 21-30 years (mean age $=23.86$ years), from the Toronto community, participated in exchange for monetary compensation. None of those who participated in the study had a history of any psychiatric illness. Consent was obtained and the rights of the participants were protected, and the guidelines of the Toronto Academic Health Science Council were upheld.

\section{Stimuli and Design}

Subjects were presented with visual and auditory stimuli. The visual stimuli were 160 pictures of famous and 160 pictures of nonfamous faces that have been used in previous studies (Hannula, Ryan, Tranel, \& Cohen, 2007; Ryan, Hannula, \& Cohen, 2007). Equal numbers of female and male stimuli were presented across the famous and nonfamous conditions. The faces were selected from a larger set of faces, all of which had associated rating scores from previous norming studies regarding the "famousness" and "nameability" of each face. Selected famous faces were those which had an average score of 3 or higher on a 5-point scale of "famousness" ( $1=$ nonfamous, $5=$ famous $)$ and an average score of 3 or higher on a 5-point scale of "nameability" ( $1=$ no associated name, $5=$ first and last names readily available). Selected nonfamous faces were those that had an average score of less than 2 on both scales. Faces were equated for brightness and placed on a 1024 by 768 black background with the eyes of each face centered in approximately the same location. The screen width measured 16.5 in. and subtended approximately $29^{\circ}$ of visual angle when participants were seated 32 in. from the screen. The faces comprised approximately $5 \mathrm{in}$. of the screen width or $9^{\circ}$ of visual angle when viewed from a distance of 32 in.

The auditory stimuli consisted of spoken names of famous and nonfamous men and women. All names were recorded by a professional voice artist. The famous names corresponded to the set of famous faces as outlined above. Nonfamous male and female names were constructed such that the first and last names of a given nonfamous name began with the same starting phoneme and had the same syllable length as a corresponding first and last names of a famous person (e.g., "Vern Kilston" was the nonfamous counterpart to "Val Kilmer"). Famous names were, on average, $1088 \mathrm{msec}$ in length $(S D=138 \mathrm{msec})$; nonfamous names were, on average, $1159 \mathrm{msec}$ in length $(S D=201 \mathrm{msec})$. The nonfamous names were uniquely associated with individual nonfamous faces.

\section{Procedure}

Prior to MEG recordings, a survey was administered to assess the subjects' familiarity with entertainment celebrities (see Appendix). Following this, a subset of the 
auditory stimuli was played backward to the subjects to detect their threshold level of hearing. Auditory stimuli were then presented at $40 \mathrm{~dB}$ above this threshold level. Visual and auditory stimuli were presented using Presentation. The MEG data collection was synchronized with the onset of visual and auditory stimulus presentation by recording the luminance of the screen and the audio sound envelope, respectively. Four blocks of stimuli were presented, two of which were composed of visual stimuli only, and two were composed of auditory stimuli only. Each block contained 80 stimuli, 40 famous and 40 nonfamous, presented in random order. Equal numbers of male/female stimuli were presented across conditions and across blocks. Subjects either saw the face or heard the name of a given famous person (and their nonfamous counterpart) during the course of the experiment; for example, participants either saw Val Kilmer's (or Vern Kilston's) face or heard his name, but not both. Half of the subjects were exposed to an auditory block first, and half were presented with a visual block at the beginning of the experiment. Subjects were asked to judge whether the presented stimulus was either male or female. Responses were made via button presses. Although successful performance of male/female judgments may require retrieval of semantic knowledge pertaining to the typicality of male/females names or the features of male versus female faces, such retrieval of general semantic knowledge should occur similarly for famous and nonfamous stimuli. Sex and recognition judgments have been dissociated through neuropsychological studies of prosopagnosic patients, (Sergent \& Villemure, 1989); and may occur through separate neural routes (Mouchetant-Rostaing, Giard, Bentin, Aguera, \& Pernier, 2000; Bruce \& Young, 1986). Sex judgments do not require the retrieval of identity or of other specific associated information related to identity; likewise, retrieval of identity does not require the categorization of sex (Wild et al., 2000; Bruce, Ellis, Gibling, \& Young, 1987). Therefore, any differences between the famous and nonfamous condition will be taken as evidence for unintentional retrieval of associated information specific to the stimulus.

\section{Data Acquisition}

All MEG data were recorded in a magnetically shielded room using a 151-channel whole-head first-order gradiometer system (VSM Medtech, Port Coquitlam, BC, Canada) with detection coils spaced $31 \mathrm{~mm}$ apart. Data acquisition was $625 \mathrm{~Hz}$. To monitor head movement, three small coils of wire were attached to the participant's head and energized with small currents before and after the task. This determined the location of the participant's head with respect to the MEG array. For each subject, a structural MRI was obtained using a 1.5-T Siemens Magnetom system at Sunnybrook's Women's Hospital.

\section{Data Analysis}

Preparation for Source Analysis

Eye-movement and blink artifacts were removed off-line from single-trial MEG data using principle components analysis (Kobayashi \& Kuriki, 1999). This approach has been shown effective for removing artifacts with amplitudes larger than the brain signals of interest (Lagerlund, Sharbrough, \& Busacker, 1997). Principal components with amplitudes larger than $1 \mathrm{pT}$ at any time in any MEG sensor were removed from the single-trial magnetic field data. Data were then averaged over 2000-msec epochs, time locked to the onset of the stimuli. The averages were low-pass filtered at $50 \mathrm{~Hz}$, and high-pass filtered at $2 \mathrm{~Hz}$. A baseline for the measured response was selected at -100 to 0 msec prior to stimulus onset. A minimum norm least squares (MNLS; L2 norm) algorithm from the Curry 5.05 software package was used to estimate the current distribution in the brain from the MEG data. A realistically shaped boundary element model for the conducting volume of the brain was extracted from individual subjects' MRIs for magnetic field computations in the MNLS analysis.

\section{Extractions of Regions of Interest}

The MNLS algorithm permits detailed modeling of distributed activity with no a priori assumptions about the number of active regions. The following procedure was used to select specific regions of interest (ROIs) from the MNLS inversion. (1) Whole-brain current flow distribution maps were thresholded at $80 \%$ of the maximum value at each time slice. Using this thresholded map, inspection of the MNLS inversions revealed that current flow in the bilateral superior temporal gyrus (STG) and the bilateral occipital cortex appeared as disjoint volumes of activation upon visual inspection (e.g., McDowell, Kissler, Berg, et al., 2005). (2) ROIs were selected that encompassed regions of high activation that overlapped the left and right STG and the left and right occipital cortex by $1 \mathrm{~cm}$, and were retained for further analysis. ROIs in and near the auditory cortex were selected from the nonfamous names condition. ROIs in and near the visual cortex were selected from the nonfamous faces condition. If sources appeared ambiguous, the threshold was raised to $90 \%$ of maximum to obtain a smaller ROI, and/or the famous names/faces condition was consulted for similar sources. (3) Source strength waveforms over time were extracted for the ROIs from the MNLS output using MatLab.

\section{Analysis of Waveform Amplitude Peaks and Latencies}

Latencies and amplitudes of evoked responses were determined from the waveforms for the first $400 \mathrm{msec}$ following stimulus onset. Amplitudes of responses were computed at each sample (1.6 msec) within the 400-msec time window. The single highest amplitude peak for this 
epoch was selected for analysis. If several peaks occurred in the waveform during this epoch, the largest was selected for analysis.

Peak amplitudes for the identical STG sources were determined for the famous and nonfamous names condition, as well as the famous and nonfamous faces condition. Similarly, peak amplitudes for identical bilateral occipital sources were determined for the famous and nonfamous faces condition, as well as the famous and nonfamous names condition. The peak amplitude of the ROIs in the famous conditions was compared to those in the nonfamous conditions within \pm 30 msec of the peak selected for the famous condition. These criteria for selecting activity peaks in MEG data for comparison across conditions are similar to those used in previous MEG studies of auditory activation (Hanlon et al., 2005; Moses et al., 2005; Thoma et al., 2003, 2005). The peak amplitudes for the famous condition were greater than or equal to three standard deviations above the mean baseline amplitude.

Sources that yielded waveforms that appeared to be extremely noisy showing no clear peaks were excluded from analysis. In addition, occasionally, sources were not found over specific regions for particular subjects. These situations resulted in three missing data points for right occipital sources in the names condition, and one right occipital source in the faces condition.

\section{Statistical Analysis}

Peak amplitudes and latencies for bilateral STG and Occipital sources found in the names and faces conditions, respectively, were examined using repeated measure analysis of variance (ANOVA). In other words, we examined auditory activity in the names condition and visual activity in the faces condition. Within-subjects factors included "source" (STG/occipital), "hemisphere" (right/ left), and "stimulus" (famous/nonfamous). Peak amplitudes and latencies for the identical auditory and visual sources found in the faces and names conditions, respectively, were also compared using similar ANOVA, that is, we examined auditory activity in the faces condition and visual activity in the names condition. Peak amplitudes of greater than two standard deviations away from the group mean for each condition were considered outliers and were removed from analysis. This resulted in the exclusion of one subject's left occipital source found for the faces condition, the same subject's right STG source found for the faces condition, and another subject's left STG source found for the faces condition.

\section{RESULTS}

\section{Familiarity with Entertainment Celebrities}

On average, subjects watched approximately 5 to $6 \mathrm{hr}$ of television per week (mean score of 3.86 on a 1-7 scale; see Appendix). Subjects viewed about two movies per month (mean score of 5.07). Celebrity magazines and Websites were read once every few months (mean scores of 3.00 and 3.36, respectively). Overall, subjects expressed a fair knowledge of celebrities (mean score of 4.21).

\section{Behavioral Responses}

Measures of response time and accuracy were analyzed in a repeated measures ANOVA with the within-subjects factors of modality type (face, name), famousness (famous, nonfamous), and sex of the presented stimulus (male, female). Due to recording error, response data were obtained for all conditions for 7 of the 10 subjects. The results that follow are for those seven subjects. A significant main effect of modality type was observed for response time $[F(1,6)=29.29, p<.01]$ but not for accuracy $(F<1)$, whereas responses were faster for the faces than for the names, responses were equivalent on accuracy across the auditory and visual conditions. There was a significant effect of fame on the speed of response $[F(1,6)=80.63, p<.001]$ and accuracy $[F(1$, $6)=27.16, p<.01]$; responses to famous stimuli were faster and more accurate than responses to the nonfamous stimuli. A significant effect of sex was also observed; responses were faster $[F(1,6)=21.05, p<.01]$ and more accurate $[F(1,6)=15.34, p<.01]$ for men than women. There was a significant interaction between modality and fame of the presented stimuli for response time $[F(1,6)=36.1, p=.001]$ and accuracy $[F(1,6)=19.1, p<.01]$; response times and accuracy were similar across famous and nonfamous faces, but responses for famous names were faster and more accurate than responses for nonfamous names. Critically, the effects of sex did not interact with the effects of fame on either measure. This suggests that subjects were responding to the sex discrimination task in a similar fashion for famous and nonfamous stimuli.

\section{Auditory Activation for Names and Visual Activation for Faces}

We examined strength and latency of the strongest peaks for STG sources in the famous and nonfamous names condition, and for occipital sources in the famous and nonfamous faces condition. Figure 1 shows sensor waveforms for the nonfamous faces and the nonfamous names conditions. Figure 2 shows the STG sources for the nonfamous names condition for a representative subject and occipital sources from the nonfamous faces condition for a representative subject, as identified using the MNLS algorithm.

The amplitude of STG activation was greater for the famous compared to the nonfamous names (see Fig- 
Figure 1. (A) Sensor waveforms for a representative subject for the nonfamous faces conditions. (B) Sensor waveforms for a representative subject for the nonfamous names condition.

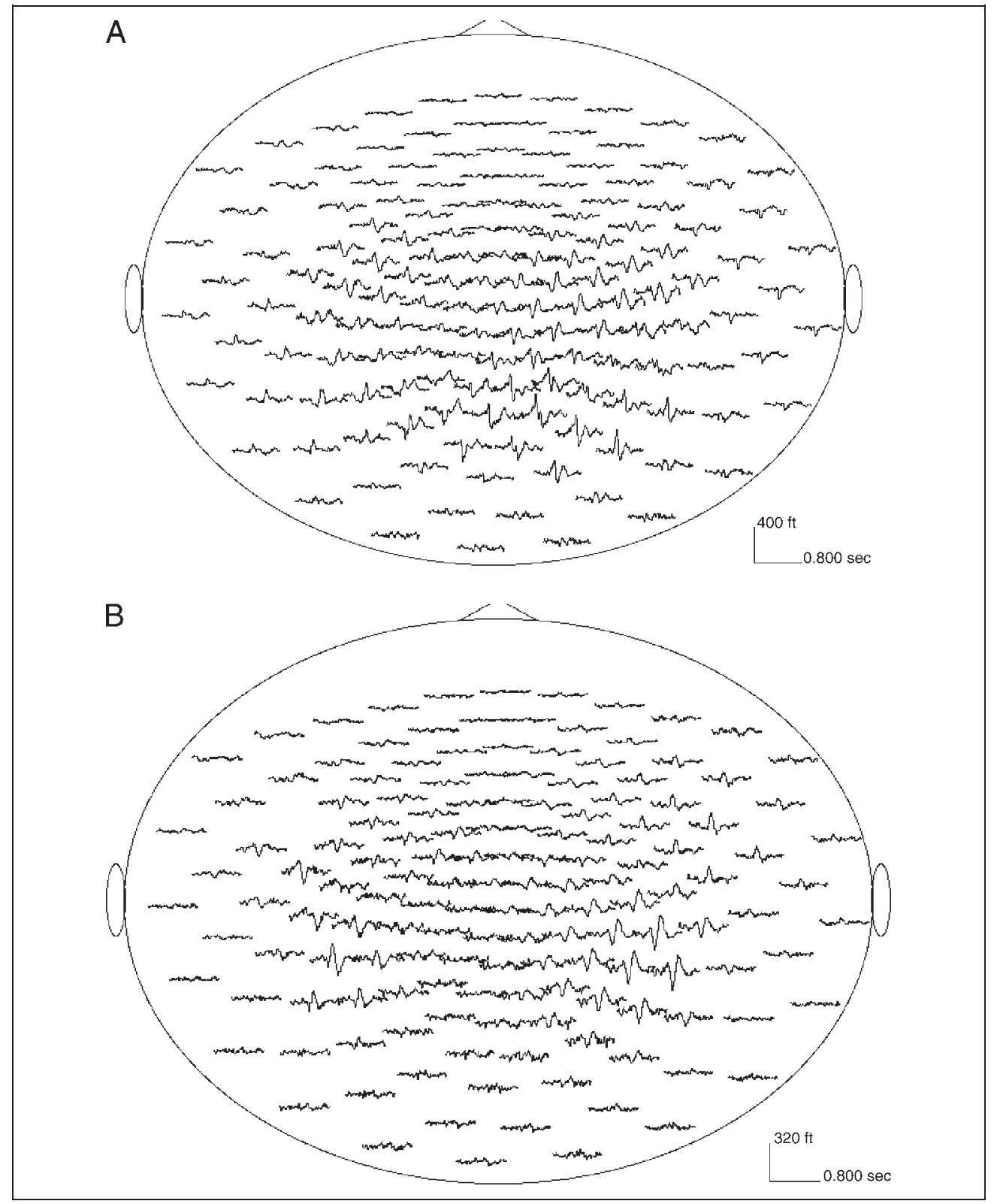

ure 3), as illustrated by a main effect of "stimulus" in planned comparisons $[F(1,9)=12.9, p=.005]$, and separate $t$ tests on the left $[t(9)=3.3, p<.01]$ and right $[t(9)=3.1, p=.01]$ hemispheres. The strongest peaks of STG activity occurred following presentation of the famous names at an average of $211 \mathrm{msec}(S D=81)$ in the left hemisphere and $204 \mathrm{msec}(S D=60)$ in the right, and following the nonfamous names at $208 \mathrm{msec}$ $(S D=88)$ in the left and $207 \mathrm{msec}(S D=59)$ in the right. No significant differences in latency were detected between the famous and nonfamous names for STG peaks.

There were no differences in amplitude of occipital peaks for the famous and nonfamous faces (see Figure 4). The strongest peaks of occipital activity occurred following presentation of the famous faces at an aver- age of 135 msec $(S D=61)$ in the left hemisphere and $117 \mathrm{msec}(S D=29)$ in the right, and following the nonfamous faces at $137 \mathrm{msec}(S D=59)$ in the left and 124 $(S D=28)$ in the right. No significant differences in latency were detected between the famous and nonfamous faces for occipital peaks. The strongest peaks in occipital sources elicited by the faces occurred earlier than the peaks in the STG sources elicited by the names $[F(1,8)=17.0, p=.003]$.

\section{Auditory Activation for Faces and Visual Activation for Names}

We examined strength and latency of the strongest peaks for STG sources in the famous and nonfamous faces 


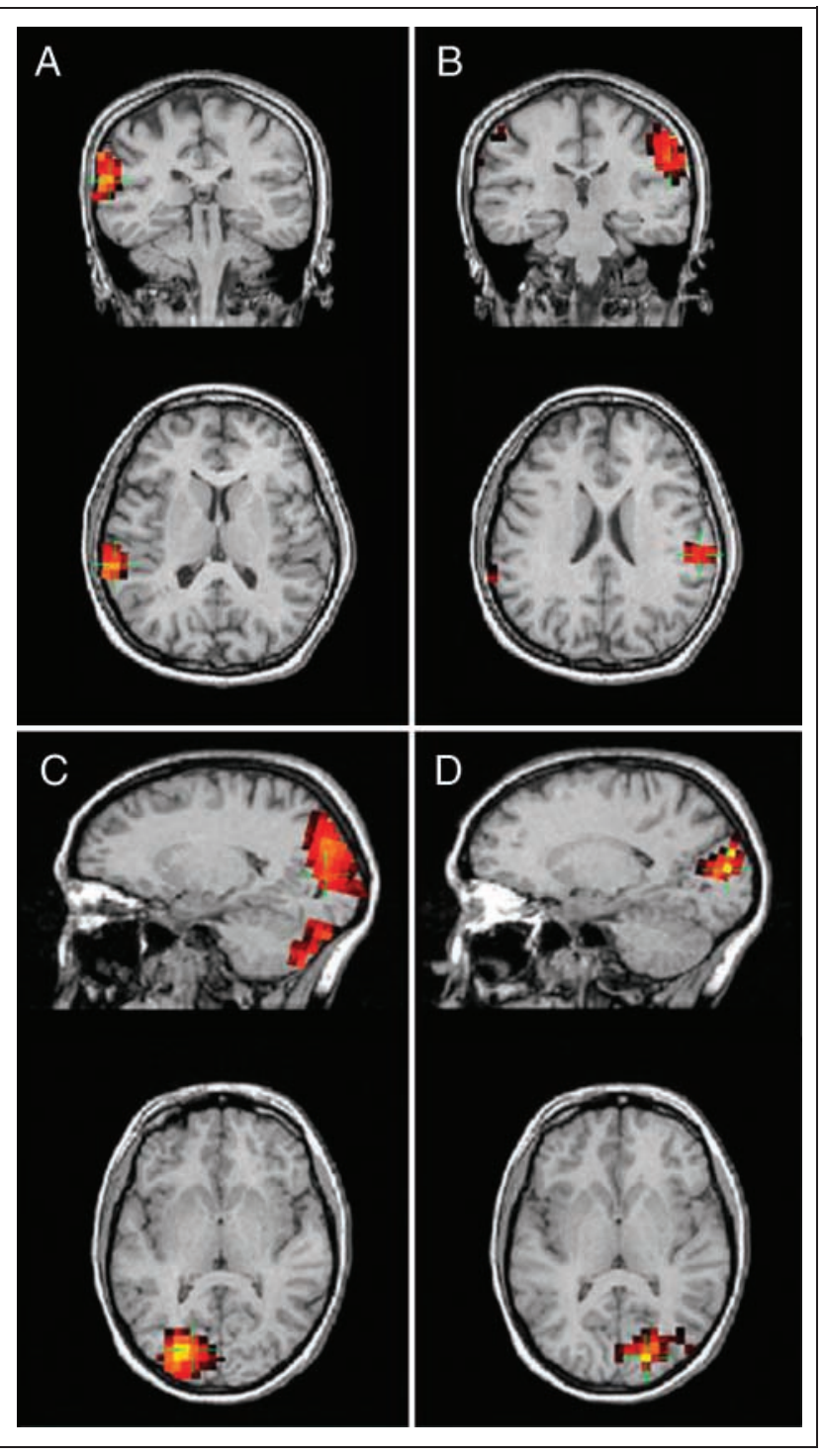

Figure 2. Auditory response to nonfamous names (top). Regions of activity obtained over the left (A) and right (B) STG using the MNLS algorithm superimposed onto an individual subject's MRI; coronal and axial slices are displayed. Visual response to nonfamous faces (bottom). Regions of activity obtained over the left (C) and right (D) occipital cortex superimposed onto an individual subject's MRI; sagittal and axial slices are displayed.

condition, and for occipital sources in the famous and nonfamous names condition.

The amplitude of STG and occipital activation was greater for the famous compared to the nonfamous conditions (see Figure 5). This effect is illustrated by a main effect of "stimulus" in the overall ANOVA $[F(1$, $5)=9.8, p<.05]$, and by a separate $t$ test on the STG [left: $t(8)=3.0, p<.05$; right: $t(8)=2.8, p<.05$ ]. Additionally, STG activation in the right hemisphere was stronger than in the left, collapsed across conditions $[F(1,7)=12.7, p<.01]$. The strongest peaks of STG activity occurred following presentation of the famous faces at an average of $201 \mathrm{msec}(S D=50)$ in the left hemisphere and $171 \mathrm{msec}(S D=56)$ in the right, and following the nonfamous faces at $195 \mathrm{msec}$ $(S D=54)$ in the left and $172 \mathrm{msec}(S D=53)$ in the right.

Amplitudes of occipital peaks were greater for the famous compared to the nonfamous names (see Figure 6), as illustrated by separate $t$ test for each hemisphere [left: $t(9)=2.8, p<.05$; right: $t(6)=2.8, p<.05$ ]. No differences in hemisphere were found for occipital peaks.

The strongest peaks of occipital activity occurred following presentation of the famous names at an average of $208 \mathrm{msec}(S D=67)$ in the left hemisphere and $201 \mathrm{msec}(S D=51)$ in the right, and following the nonfamous names at $210 \mathrm{msec}(S D=68)$ in the left and 198 msec $(S D=48)$ in the right. No significant differences in latency were detected between the famous and nonfamous conditions for STG or occipital peaks when responses to faces and names were combined. However, when the latencies for the strongest peak within occipital and STG regions were examined separately

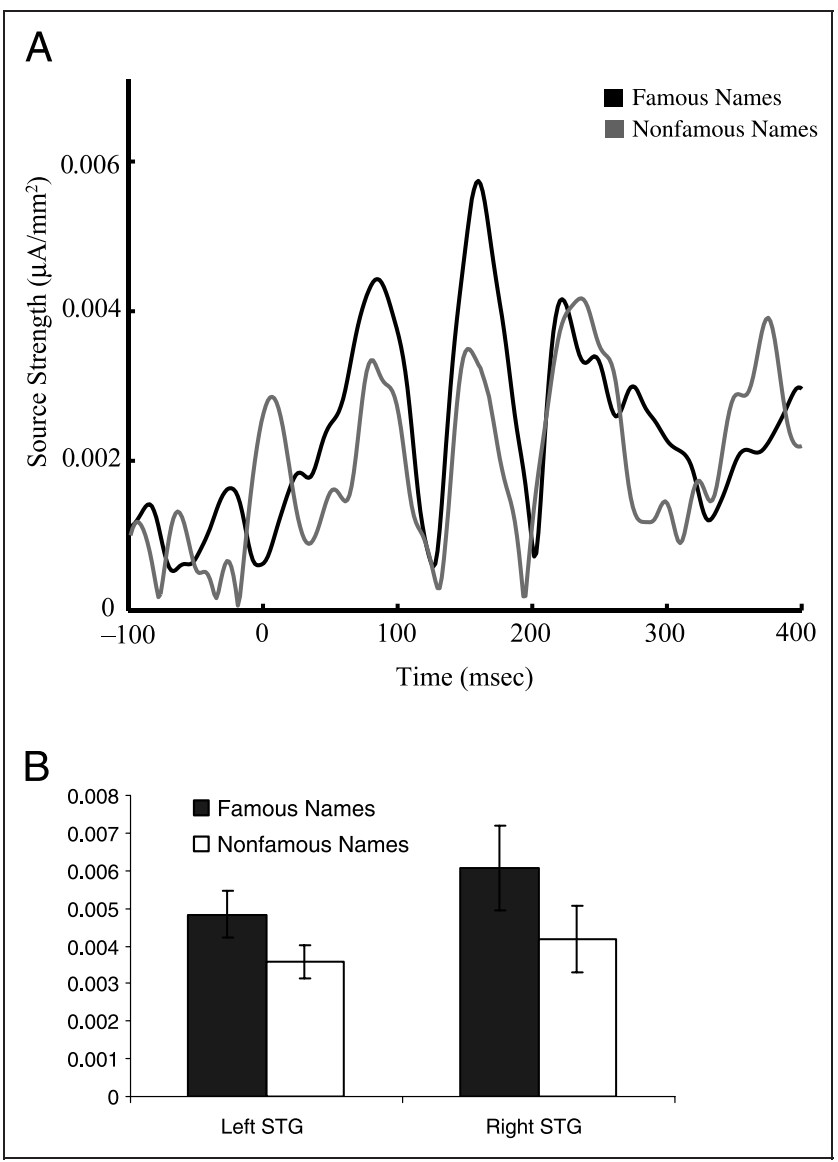

Figure 3. (A) Strength of response over time to famous and nonfamous names for a source overlying the left STG in a representative participant. (B) Strength of peak activation from left and right STG sources following presentation of famous and nonfamous names averaged across participants. Peak amplitude of STG activation was greater for the famous compared to the nonfamous names. 


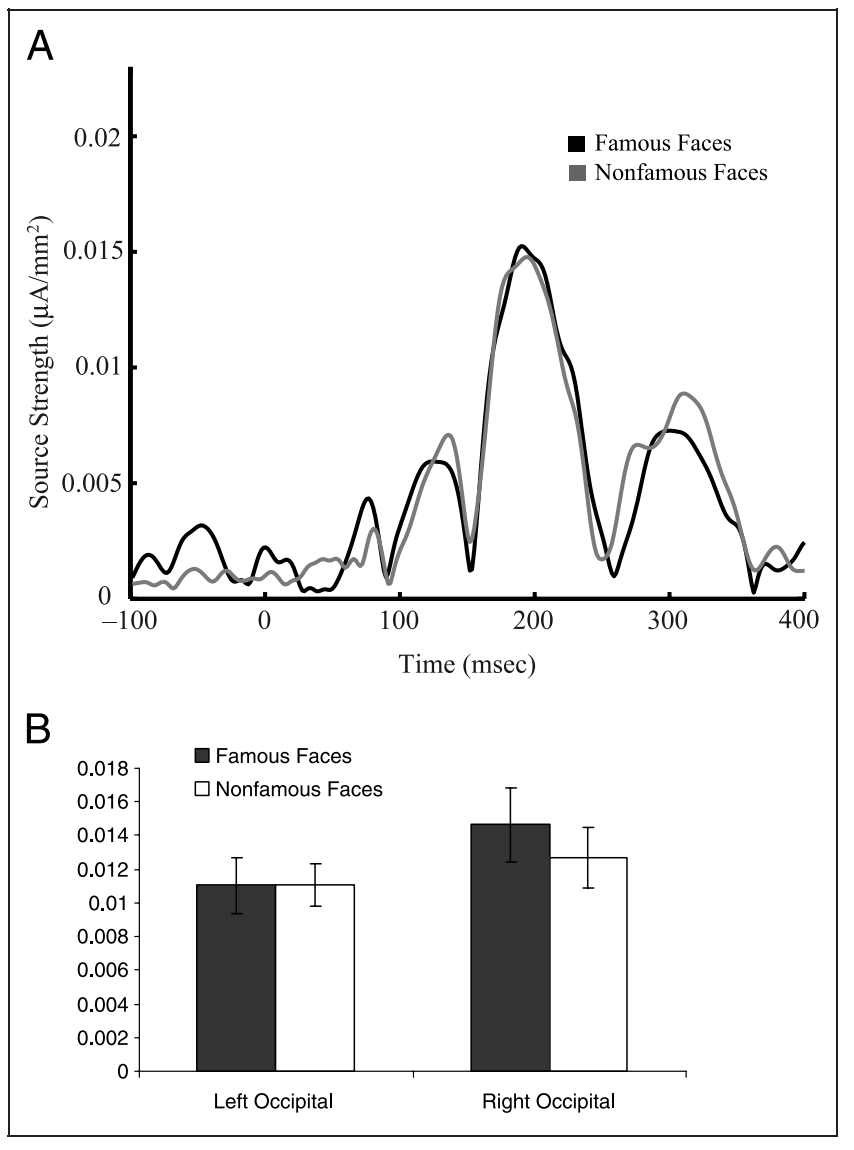

Figure 4. (A) Strength of response over time to famous and nonfamous faces for a source overlying the left occipital cortex in a representative participant. (B) Strength of peak activation from left and right occipital cortex sources following presentation of famous and nonfamous faces averaged across participants. There were no differences in amplitude of occipital peaks for the famous and nonfamous faces.

for the names and faces conditions, there was a nonsignificant effect of "source" for the names condition $[F(1,6)=1.33, p>.25]$, but a significant effect of "source" for the faces condition $[F(1,8)=7.18, p<.05]$. Therefore, the strongest peak of activity within STG and occipital regions occurred at similar latency for the names, but latency of the strongest peak in the occipital cortex preceded that from the STG when faces were presented.

\section{DISCUSSION}

Our prior experiences alter our perceptions. Notably, responses within the auditory cortex were larger for visually presented famous versus nonfamous faces. Likewise, activation within the visual cortex was larger for auditorily presented famous versus nonfamous names. The cross-modal activity pattern seen here is not unlike that found in synesthetic individuals in whom the phenomenon does not depend on experience (Ramachandran \& Hubbard, 2001). Here, these responses were likely driven by information that, through experience, had come to be associated with the presented item, such as the name or occupation that accompanies a particular face. We observed these effects early during processing, within 150-250 msec, which is within the time window during which largely perceptual processes are thought to occur as opposed to a later time window during which conceptual/semantic processes and/or the retrieval of associated information are largely purported to occur (e.g., Itier et al., 2006; Schweinberger et al., 2002; Donaldson \& Rugg, 1998, 1999).

Our findings not only add to the growing claims that the neural regions that are on-line during processing are altered through previous experience (Prince, Daselaar, \& Cabeza, 2005), but our work further suggests that such changes are obligatory. Previous work that outlined the changes in neural activation during processing as a function of prior learning explicitly required subjects to

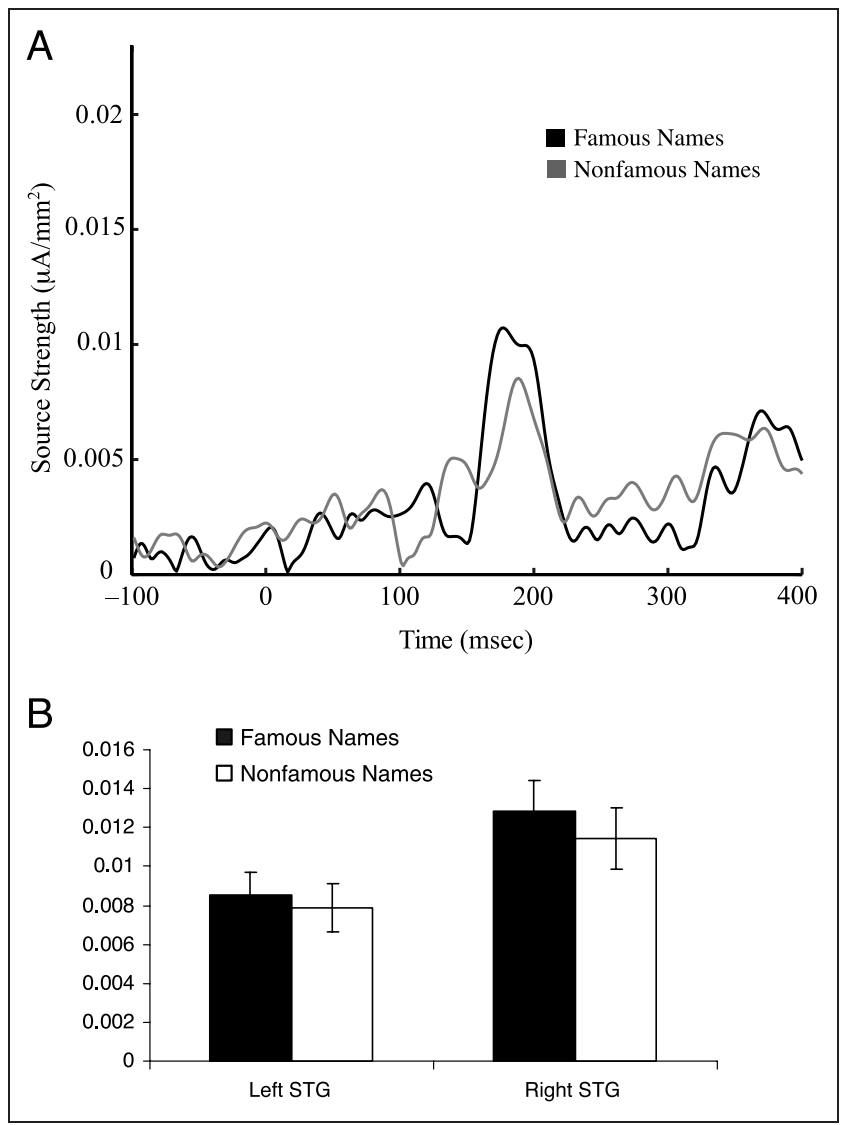

Figure 5. (A) Strength of response over time to famous and nonfamous faces for a source overlying the left STG in a representative participant. (B) Strength of peak activation from left and right STG sources following presentation of famous and nonfamous faces averaged across participants. Peak amplitude of STG activation was greater for the famous compared to the nonfamous faces. 


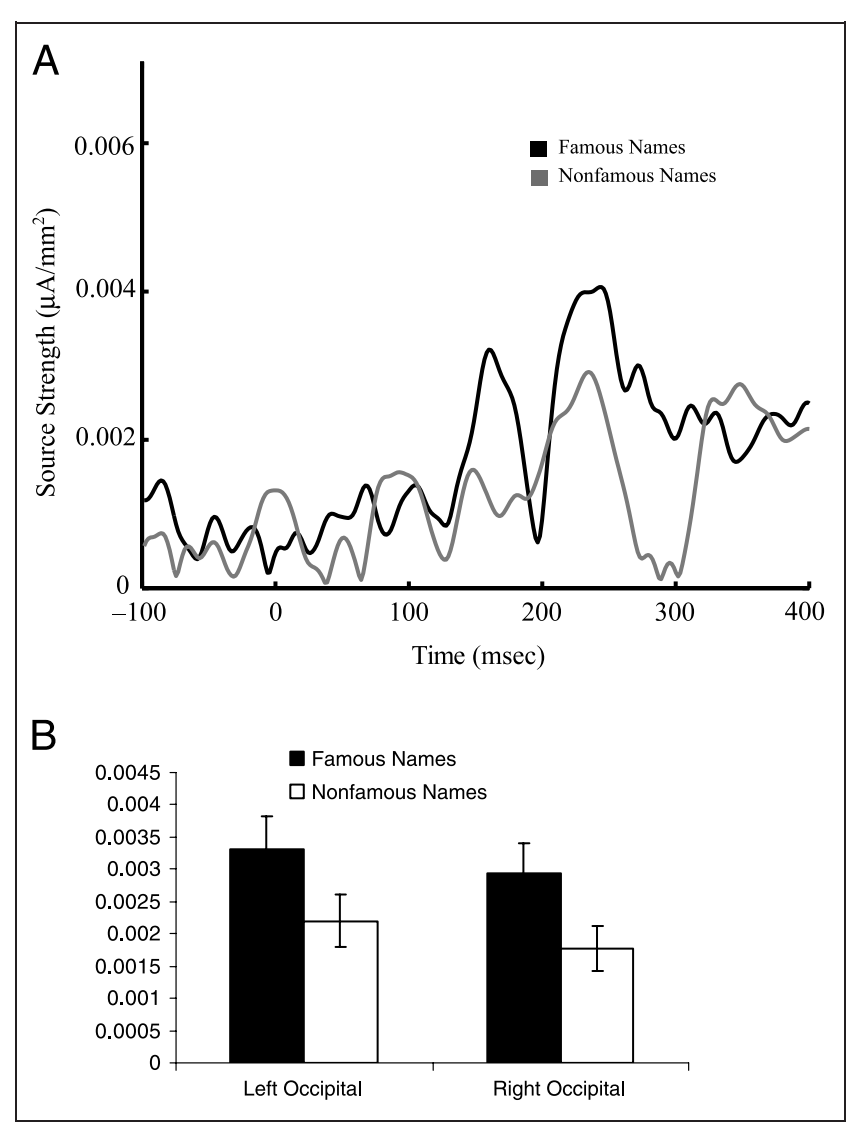

Figure 6. (A) Strength of response over time to famous and nonfamous names for a source overlying the left occipital cortex in a representative participant. (B) Strength of peak activation from left and right occipital cortex sources following presentation of famous and nonfamous names averaged across participants. Peak amplitude of occipital activation was greater for the famous compared to the nonfamous names.

access their memories and/or to use any remembered associated information to aid in the recognition memory judgments (Nyberg et al., 2000; Wheeler et al., 2000). However, in our study, associated information was not present during the experimental session in the current work and was not needed to aid in task performance. Thus, the differences in neural responses between the famous and nonfamous stimuli were observed even though we did not require subjects to remark on the contents of their memories. As such, we propose that prior experiences not only influence processing but that they do so in a compulsory manner. Below, we describe the modulation of neural responses within the STG and the occipital cortex as a function of prior learning in the context of a variety of findings, from visual imagery to word meaning, which ultimately serves to address the fundamental question of how our prior experiences may fundamentally alter our perceptions.

Here, neural responses within the STG were modulated by prior learning. This is consistent with Saykin et al. (1999), who demonstrated that previously studied auditorily presented words elicited greater activation, as revealed by fMRI, within the STG compared to novel words. Such modulation of responses is apparent early in processing; here, peak differences were observed between famous and nonfamous names in the STG approximately $200 \mathrm{msec}$ following stimulus onset. These latency findings are consistent with other MEG findings from Marinkovic et al. (2003) and Simos, Breier, Zouridakis, and Papanicolaou (1998). Simos et al. (1998) observed neural responses to a continuous recognition task of spoken words localized to the STG that arose within 150-200 msec of stimulus onset. Marinkovic et al. found differential responses within the superior temporal plane for repeated versus novel words approximately 225 msec following stimulus onset. Therefore, in these previous studies and the current work, auditory responses distinguished previously learned (famous names) from novel (nonfamous names) auditory stimuli early in processing.

Although the auditory names were not presented along with the visual faces, neural responses within the STG were also significantly greater for famous faces compared to nonfamous faces. This is consistent with the work from Nyberg et al. (2000) and Wheeler et al. (2000) which demonstrated activation in auditory regions upon presentation of a lone visual stimulus which had been previously associated with an auditory item. It is not unreasonable to expect activation in neural regions associated with auditory processing as a function of prior learning, even in the absence of an acoustic stimulus, when findings from auditory imagery are considered. For instance, Kraemer, Macrae, Green, and Kelley (2005) had subjects passively listen to familiar and unfamiliar excerpts of music in which a silent gap was inserted. Greater activation was observed in the primary auditory cortex and auditory association regions during the silent gap of familiar versus unfamiliar songs. Altogether, responses in the auditory cortex may be revealed during processing of a visual target item if that visual item had been associated with auditory information during a prior learning event.

Responses within the occipital cortex did not distinguish famous from nonfamous faces. Nevertheless, the findings here of increased neural responses to famous compared to nonfamous names within the occipital cortex harken back to findings from work on visual imagery, and suggest that neural activity within the occipital cortex can be affected through prior experience. Kosslyn, Thompson, Kim, and Alpert (1995) demonstrated increased activation in the primary visual cortex as revealed by positron emission tomography when subjects were asked to visualize previously studied objects in response to an auditory prompt. From this, Kosslyn et al. (1995) suggested that although primary visual areas may not be the site of storage for visual representations, higher-level areas which are involved in the storage of visual information may induce differential activity in these primary sensory 
regions. Therefore, neural activity in the occipital cortex, like the STG, may be altered due to prior experience and stored information.

Our findings of neural activation within the occipital cortex approximately 150-200 msec following stimulus onset of famous or nonfamous faces are consistent with Iidaka, Matsumoto, Haneda, Okada, and Sadato (2006), who demonstrated through fMRI and ERP experiments conducted on the same group of subjects that the N170, a negative deflection in ERP occurring approximately 170 msec (140-200 msec; Itier et al., 2006) following the onset of face stimuli, is correlated with hemodynamic responses within occipital cortices. Our findings of larger neural responses for famous versus nonfamous names, at approximately $200 \mathrm{msec}$ following the onset of the name, suggest that the famous name had become associated with visual information, presumably a face, through prior learning episodes. When considered with the imagery findings mentioned above, we find that the activation observed in the occipital cortex is altered due to past experiences.

It should be noted that famous and nonfamous names each elicited some activation within the occipital cortex, and that famous and nonfamous faces each elicited some activation within the STG. This may be surprising as one does not expect visual information to be associated with nonfamous names; likewise, auditory/verbal information is not assumed to be associated with nonfamous faces. In the current paradigm, famous and nonfamous stimuli were intermixed, thereby likely prompting subjects to expect famous and nonfamous stimuli; thus, we may be observing initial "false fame" responses to the nonfamous stimuli. The nonfamous faces were obtained from magazines and catalogs and therefore appear similar in picture quality to pictures of famous people, or perhaps resemble other people the subjects had encountered in real life. Also, the names were controlled such that the initial phonemes of the first and last names were the same across famous and nonfamous stimuli. In fact, results from a subsequent behavioral paradigm, in which subjects were played either a 200-msec truncated or full version of the famous and nonfamous name stimuli used here, revealed that previously stored memories were accessed even during the presentation of nonfamous names (Ryan, Moses, Ostreicher, \& Riggs, in preparation). Although subjects classified famous truncated names as "famous" more often than the nonfamous counterparts, subjects were still more likely to classify the nonfamous truncated names as "famous" than "nonfamous," and even generated a famous name to the nonfamous truncated names on over half of the trials when asked to guess what the full name might have been. By contrast, full versions of the names were accurately classified as famous/nonfamous. Therefore, the nonfamous stimuli (regardless of whether they are faces or names) in the current paradigm may have inadvertently activated a search through a similar set of representations of famous (or otherwise, known) people which are then perhaps not maintained in an active state upon further processing of the auditory or visual stimuli. Nevertheless, the responses observed in the occipital cortex were larger for famous versus nonfamous names, and the responses observed in the STG were larger for famous versus nonfamous faces, suggesting that our prior experiences alter the responses within associated neural regions that are observable early in processing.

Although it is clear that processing is modified through prior learning, a question remains regarding how (or even whether) associated information is exerting its influence on perception. In particular, do the present findings reflect the cortical reactivation of separate, but associated, information, or is perception itself fundamentally altered through experience? The standard view has proposed that through prior learning episodes, associated information may be added as a separate link to the target item, without necessarily affecting the nature of the representation of the target item itself (Anderson, 1976). Indeed, the current work could be taken to support the notion that the processing of an item results in the rapid access and activation of distinct, but associated information, through previously established links or connections. However, processing was affected early by associated information. In particular, prior experiences altered neural responses during a time at which it is hypothesized that the perceptual representation is formed (Roisson et al., 2000; Bentin, Allison, Puce, Perez, \& McCarthy, 1996). It should be noted that there are indications that conceptual or identification processes could occur to some extent within this time window as well (Jemel, Pisani, Calabria, Crommelinck, \& Bruyer, 2003; Itier \& Taylor, 2002). Thus, although we speculate here that prior experiences may alter perception itself, further work remains to rule out the contribution of conceptual/semantic processes to the observed effects.

Further evidence from the current work speaks to the notion that perception may be fundamentally altered through prior experience. We found no differences in the peak latency of responses between the STG and the occipital cortex for auditory names. When considered in isolation, this finding would suggest that rather than the auditory stimulus reactivating associated visual information, the representation of the famous person encompasses both the visual and auditory information within a single representation. However, upon presentation of a visual face, the peak latency within the occipital cortex occurred earlier than the peak response from the STG. This may be taken to support the alternate account noted above that presentation of an item activates distinct, associated information early in processing.

It would appear that the latency findings from faces versus names may be at odds with one another, each 
supporting an alternate account of how past experience alters current perceptions. However, recent work by Bar et al. (2006) and Bar (2003, 2004) may shed some light on this issue. In short, Bar and colleagues suggest that, upon presentation of a visual stimulus, information regarding low-level spatial frequencies is rapidly projected from early visual regions to the orbito-frontal cortex. From this sparse information, the orbito-frontal cortex activates a small set of representations that could serve to match the perceptual input, thereby facilitating eventual recognition. In that case, the early response seen here within the occipital cortex for the faces may reflect the projection of low spatial frequency information to frontal regions, whereas the subsequent response for famous faces within the STG may reflect the activation of the representation in memory that matches the perceptual input. In this case, the representation of the object (face or name) may include other information that had been present during the previous learning episode and serves to distinguish the object from other items, either novel or familiar.

A similar phenomenon may be occurring for the names as well (Marinkovic, 2004; Gold \& Buckner, 2002), even though there were no detectable differences in the peak latency between STG and occipital responses for the famous names. Although there is a clear and consistent onset of visual information for the presented faces, the "onset" of auditory information is less discrete. Although the first phonemes were the same across famous and nonfamous names, the duration of the first phoneme varied from trial to trial across the names, thereby potentially reducing our signal-to-noise ratio for the mean STG response. Although timing differences exist between the occipital cortex and the STG for faces (and possibly even for names), the auditory and visual information may, nonetheless, be combined into a representation of the famous person that is activated upon processing of initial, sparse information. Thus, the current findings support the account that suggests that an object is composed of more than what it imparts upon the senses.

This notion receives converging support from recent findings on word meaning. For instance, Pulvermuller, Shytrov, and Ilmoniemi (2005) presented subjects with action words and pseudowords, whereas neural responses were recorded with MEG. The action words referred to those that could either be performed with the face (e.g., eat) or the leg (e.g., kick). Action words, relative to the pseudowords, elicited somatotopic activity in the motor cortex. Such activity was evident within 170-200 msec following the onset of the second syllable which, due to the design of the stimuli, was the first moment when the action words could be disambiguated from the pseudowords. Likewise, reading words that depict odors not only activates the same neural regions that are related to language processing as does reading neutral words but reading odor words also activates regions within the olfactory cortex (Gonzalez et al., 2006). Gonzalez et al. (2006) and Pulvermuller et al. (2005) interpreted their findings as suggesting that words are processed by distributed cell assemblies or systems, including those that are involved in processing associated semantic and sensory information; that is, one cannot read, or perceive, the word, without automatically and obligatorily accessing its meaning. An alternate interpretation proposed by James and Gauthier (2003) suggests that semantic information is stored in what are considered to be largely perceptual regions. Similar to that position, findings of increased activation in early visual association cortices, as revealed through positron emission tomography, in response to naming auditory stimuli, led Tranel et al. (2003) to argue that such activation may reflect the retrieval of conceptual knowledge that is pertinent to the task at hand. With respect to the current work, we suggest that the above studies indicate that our prior experiences may change our stored representations, leading to an altered perceptual experience. The "meaning" of the famous face, for example, is found not only in the visual information but also in the information that has come to be associated with it over time (e.g., name, occupation). That is, the identification and semantic analysis of an item may be achieved through evaluation of the associated information that has altered, and is present within, the perceptual representation.

In this sense, experience provides a sort of perceptual plasticity by which the processing of an item is everchanging. It may be these modifications that are enacted upon the stored representation that allow one to distinguish a previously encountered item from a novel one. That is, perceptual plasticity may induce the requisite changes to a maintained representation that are needed in order to accurately judge whether a presented item has been previously encountered, whether it is by modifying the existing representation of the item, or linking distinct, associated information to that item representation. In either event, the onset of stronger responses within additional sensory modalities, other than the modality in which the external stimulus is presented, may feed-forward to higher-order brain regions, and culminate in neuronal markers of familiarity/ recognition that typically occur around 250-600 msec, as revealed through electroencephalogram/ERP and MEG studies (Marinkovic et al., 2003; Paller et al., 2003).

The notion of perceptual plasticity - that prior learning affects changes in or to the representation of a target item-makes contact with William James' (1890) assertion that perception of an item is a combination of what is externally presented and what is associated with that item within the brain/mind. Regardless of how memory exerts its influence on perception, it is clear that (to borrow from Heraclitus) just as we cannot step twice into the same river, we cannot perceive twice the same form. 


\section{APPENDIX}

(1) On average, how many hours of television do you watch per week?

1

0 hours
1-2 hours

3-4 hours

5-6 hours
5

$7-8$ hours
6

9-10 hours
7

$10+$ hours

(2) How often do you go to the movies/rent a movie/watch a movie on TV?

never
2
4

once a year every few months once a month
5

twice a month once a week a few times per week

(3) How often do you read celebrity/fashion magazines?

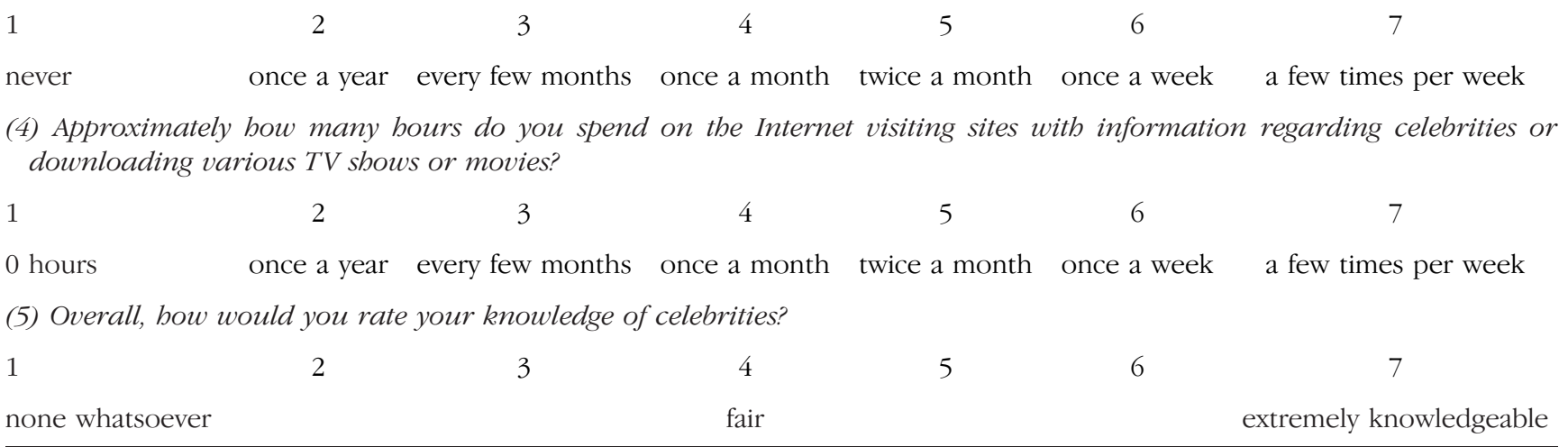

\section{Acknowledgments}

We thank Guy Earle for creating the auditory stimuli. We also thank Bernhard Ross for comments and assistance with artifact rejection. This work was supported by grants from the Natural Sciences and Engineering Research Council of Canada (J. D. R., E. T.) and the Canada Chairs Program (J. D. R.).

Reprint requests should be sent to Jennifer D. Ryan, Rotman Research Institute, Baycrest, 3560 Bathurst St., Toronto, Ontario, Canada M6A 2E1, or via e-mail: jryan@rotman-baycrest.on.ca.

\section{REFERENCES}

Anderson, J. R. (1976). Language, memory and thought. Hillsdale, NJ: Erlbaum

Bar, M. (2003). A cortical mechanism for triggering top-down facilitation in visual object recognition. Journal of Cognitive Neuroscience, 15, 600-609.

Bar, M. (2004). Visual objects in context. Nature Reviews Neuroscience, 5, 617-629.

Bar, M., Kassam, K. S., Ghuman, A. S., Boshyan, J., Schmidt, A. M., Dale, A. M., et al. (2006). Top-down facilitation of visual recognition. Proceedings of the National Academy of Sciences, 103, 449-454.

Bentin, S., Allison, T., Puce, A., Perez, E., \& McCarthy, G. (1996). Electrophysiological studies of face perception in humans. Journal of Cognitive Neuroscience, 8, 551-565.

Bruce, V., Ellis, H. D., Gibling, F., \& Young, A. W. (1987). Parallel processing of the sex and familiarity of faces. Canadian Journal of Psychology, 41, 510-520.

Bruce, V., \& Young, A. W. (1986). Understanding face recognition. British Journal of Psychology, 77, 305-327.

Donaldson, D. I., \& Rugg, M. D. (1998). Recognition memory for new associations: Electrophysiological evidence for the role of recollection. Neuropsychologia, 36, 377-395.
Donaldson, D. I., \& Rugg, M. D. (1999). Event-related potential studies of associative recognition and recall:

Electrophysiological evidence for context dependent retrieval processes. Cognitive Brain Research, 8, 1-16.

Gold, B. T., \& Buckner, R. L. (2002). Common prefrontal regions coactivate with dissociable posterior regions during controlled semantic and phonological tasks. Neuron, 35, 803-812.

Gonzalez, J., Barros-Loscertales, A., Pulvermuller, F., Meseguer, V., Sanjuan, A., Belloch, V., et al. (2006). Reading cinnamon activates olfactory brain regions. Neuroimage, 32, 906-912.

Hanlon, F. M., Miller, G. A., Thoma, R. J., Irwin, J. G., Jones, A. Moses, S. N., et al. (2005). Distinct M50 and M100 auditory gating deficits in schizophrenia. Psychophysiology, 42, 417-427.

Hannula, D. E., Ryan, J. D., Tranel, D., \& Cohen, N. J. (2007). Rapid onset relational memory effects are evident in eye movement behavior, but not in hippocampal amnesia. Journal of Cognitive Neuroscience, 19, 1690-1705.

Iidaka, T., Matsumoto, A., Haneda, K., Okada, T., \& Sadato, N. (2006). Hemodynamic and electrophysiological relationship involved in human face processing: Evidence from a combined fMRI-ERP study. Brain and Cognition, 60, 176-186.

Itier, R. J., Herdman, A. T., George, N., Cheyne, D., \& Taylor, M. (2006). Inversion and contrast-reversal effects on face processing assessed by MEG. Brain Research, 1115, 108-120.

Itier, R. J., \& Taylor, M. J. (2002). Inversion and contrast polarity reversal affect both encoding and recognition processes of unfamiliar faces: A repetition study using ERPs. Neuroimage, 21, 1518-1532.

James, T. W., \& Gauthier, I. (2003). Auditory and action semantic features activate sensory-specific perceptual brain regions. Current Biology, 13, 1792-1796.

James, W. (1983). The principles of psychology. Cambridge: Harvard University Press. (Original work published 1890.) 
Jemel, B., Pisani, M., Calabria, M., Crommelinck, M., \& Bruyer, R. (2003). Is the N170 for faces cognitively penetrable? Evidence from repetition priming of Mooney faces of familiar and unfamiliar persons. Brain Research, Cognitive Brain Research, 17, 431-446.

Kobayashi, T., \& Kuriki, S. (1999). Principal component elimination method for the improvement of $S / N$ in evoked neuromagnetic field measurements. IEEE Transactions on Biomedical Engineering, 46, 951-958.

Kosslyn, S. M., Thompson, W. L., Kim, I. J., \& Alpert, N. M. (1995). Topographical representations of mental images in primary visual cortex. Nature, 378, 496-498.

Kraemer, D. J. M., Macrae, C. N., Green, A. E., \& Kelley, W. M. (2005). Sound of silence activates auditory cortex. Nature, 434, 158.

Lagerlund, T. D., Sharbrough, F. W., \& Busacker, N. E. (1997). Spatial filtering of multichannel electroencephalographic recordings through principal component analysis by singular value decomposition. Journal of Clinical Neurophysiology, 14, 73-82.

Marinkovic, K. (2004). Spatiotemporal dynamics of word processing in the human cortex. The Neuroscientist, 10, $142-152$.

Marinkovic, K., Dhond, R. P., Dale, A. M., Glessner, M., Carr, V., \& Halgren, E. (2003). Spatiotemporal dynamics of modalityspecific and supramodal word processing. Neuron, 38, 487-497.

McDowell, J. E., Kissler, J. M., Berg, P., Dyckman, K. A., Gao, Y., Rockstroh, B., et al. (2005). Electroencephalography/ magnetoencephalography study of activities preceding prosaccades and antisaccades. NeuroReport, 16, 663-668.

Moses, S. N., Houck, J. M., Martin, T., Hanlon, F. M., Ryan, J. D., Thoma, R. J., et al. (2007). Dynamic neural activity recorded from human amygdala during fear conditioning using magnetoencephalography. Brain Research Bulletin, 71, 452-460.

Moses, S. N., Martin, T., Houck, J. M., Ilmoniemi, R. J., \& Tesche, C. D. (2005). The C50m response: Conditioned magnetocerebral activity recorded from the human brain. Neuroimage, 27, 778-788.

Mouchetant-Rostaing, Y., Giard, M.-H., Bentin, S., Aguera, P.-E., \& Pernier, J. (2000). Neurophysiological correlates of face gender processing in humans. European Journal of Neuroscience, 12, 303-310.

Nyberg, L., Habib, R., McIntosh, A. R., \& Tulving, E. (2000). Reactivation of encoding-related brain activity during memory retrieval. Proceedings of the National Academy of Sciences, 97, 1120-1124.

Paller, K. A., Ranganath, C., Gonsalves, B., LaBar, K. S., Parrish, T. B., Gitelman, D. R., et al. (2003). Neural correlates of person recognition. Learning and Memory, 10, 253-260.

Pickering, E. C., \& Schweinberger, S. R. (2003). N200, N250r, and N400 event-related brain potentials reveal three loci of repetition priming for familiar names. Journal of Experimental Psychology: Learning, Memory, and Cognition, 6, 1298-1311.
Prince, S. E., Daselaar, S. M., \& Cabeza, R. (2005). Neural correlates of relational memory: Successful encoding and retrieval of semantic and perceptual associations. Journal of Neuroscience, 25, 1203-1210.

Pulvermuller, F., Shytrov, Y., \& Ilmoniemi, R. (2005). Brain signatures of meaning access in action word recognition. Journal of Cognitive Neuroscience, 17, 884-892.

Ramachandran, V., \& Hubbard, E. M. (2001). Synaesthesia-A window into perception thought and language. Journal of Consciousness Studies, 8, 3-34.

Roisson, B., Gauthier, I., Tarr, M. J., Despland, P., Bruyer, R., Linotte, S., et al. (2000). The N170 occipito-temporal component is delayed and enhanced to inverted objects: An electrophysiological account of face-specific processes in the human brain. NeuroReport, 11, 69-74.

Ryan, J. D., Hannula, D. E., \& Cohen, N. J. (2007). The obligatory effects of memory on eye movements. Memory, 15, 508-525.

Ryan, J. D., Moses, S. N., Ostreicher, M., \& Riggs, L. (in preparation). Accessibility of stored memory representations.

Saykin, A. J., Johnson, S. C., Flashman, L. A., McAllister, T. W., Sparling, M., Darcey, T. M., et al. (1999). Functional differentiation of medial temporal and frontal regions involved in processing novel and familiar words. Brain, 122, 1963-1971.

Schweinberger, S. R., Pickering, E. C., Burton, A. M., \& Kaufmann, J. M. (2002). Human brain potential correlates of repetition priming in face and name recognition. Neuropsychologia, 40, 2057-2073.

Sergent, J., \& Villemure, J. G. (1989). Prosopagnosia in a right hemispherectomized patient. Brain, 12, 975-995.

Simos, P. G., Breier, J. J., Zouridakis, G., \& Papanicolaou, A. C. (1998). Identification of language-specific brain activity using magnetoencephalography. Journal of Clinical and Experimental Neuropsychology, 20, 706-722.

Thoma, R. J., Hanlon, F. M., Moses, S. N., Edgar, J. C., Huang, M., Weisend, M. P., et al. (2003). Lateralization of auditory sensory gating and neuropsychological dysfunction in schizophrenia. American Journal of Psychiatry, 160, 1595-1605.

Thoma, R. J., Hanlon, F. M., Moses, S. N., Ricker, D., Huang, M., Edgar, C., et al. (2005). M50 sensory gating predicts negative symptoms in schizophrenia. Schizophrenia Research, 73, 311-318.

Tranel, D., Damasio, H., Eichhorn, G. R., Grabowski, T., Ponto, L. L. B., \& Hichwa, R. D. (2003). Neural correlates of naming animals from their characteristic sounds. Neuropsychologia, 41, 847-854.

Wheeler, M. E., Petersen, S. E., \& Buckner, R. L. (2000). Memory's echo: Vivid remembering reactivates sensoryspecific cortex. Proceedings of the National Academy of Sciences, 97, 1125-1129.

Wild, H. A., Barrett, S. E., Spence, M. J., O’Toole, A. J., Cheng, Y. D., \& Brooke, J. (2000). Recognition and sex categorization of adults' and children's faces: Examining performance in the absence of sex-stereotyped cues. Journal of Experimental Child Psychology, 77, 269-291. 